\title{
Effect of IL-6 and hsCRP Serum Levels on Functional Prognosis in Stroke Patients Undergoing IV-Thrombolysis: Retrospective Analysis
}

This article was published in the following Dove Press journal: Clinical Interventions in Aging

\author{
Hanna Pawluk (D) \\ Grzegorz Grześk ${ }^{2}$ \\ Renata Kołodziejska (1D \\ Mariusz Kozakiewicz (D) \\ Alina Woźniak' \\ Elżbieta Grzechowiak ${ }^{4}$ \\ Maciej Szumny ${ }^{4}$ \\ Piotr Sobolewski $\mathbb{D}^{5}$ \\ Leszek Bieniaszewski $\mathbb{1 D}^{6}$ \\ Grzegorz Kozera $\mathbb{D}^{6}$ \\ 'Department of Medical Biology and \\ Biochemistry, Faculty of Medicine, \\ Collegium Medicum in Bydgoszcz, \\ Nicolaus Copernicus University in Toruń, \\ Bydgoszcz, Poland; ${ }^{2}$ Department of \\ Cardiology and Clinical Pharmacology, \\ Collegium Medicum in Bydgoszcz, \\ Nicolaus Copernicus University in Toruń, \\ Bydgoszcz, Poland; ${ }^{3}$ Department of \\ Geriatrics, Division of Biochemistry and \\ Biogerontology, Collegium Medicum in \\ Bydgoszcz, Nicolaus Copernicus \\ University in Toruń, Bydgoszcz, Poland; \\ ${ }^{4}$ Department of Neurology, Faculty of \\ Medicine, Collegium Medicum in \\ Bydgoszcz, Nicolaus Copernicus \\ University in Toruń, Bydgoszcz, Poland; \\ ${ }^{5}$ Department of Neurology and Stroke \\ Unit, Holy Spirit Specialist Hospital in \\ Sandomierz, Jan Kochanowski University, \\ Kielce, Poland; ${ }^{6}$ Medical Stimulation \\ Center, Medical University of Gdańsk, \\ Gdańsk, Poland
}

Correspondence: Hanna Pawluk Department of Medical Biology and Biochemistry, Faculty of Medicine, Collegium Medicum in Bydgoszcz, Nicolaus Copernicus University in Toruń, Karłowicza 24, Bydgoszcz 85-092, Poland Tel +48525853755

Email hannapawluk।@wp.pl
Purpose: We evaluated the relationship between pretreatment IL-6 and hsCRP levels, symptom severity and functional outcome of patients with acute ischemic stroke (AIS) treated with IV-thrombolysis.

Patients and Methods: IL-6 and hsCRP samples were obtained from 83 consecutively treated Caucasian patients with AIS prior to initiation of IV-thrombolysis. Severity of stroke symptoms was assessed using the National Institutes of Health Stroke Scale (NIHSS), whereas functional outcome was assessed with modified Rankin Scale (mRS). The commercially available sets of enzymatic immune tests were used to estimate the concentrations of inflammatory markers in serum.

Results: Medians of IL-6 serum concentrations prior to IV-thrombolysis were lower in patients with favorable (mRS 0-2 pts) functional outcome than in those with unfavorable (mRS 3-6 pts) functional outcome, both at hospital dismission (5.92: 2.30-7.71 vs 9.46: $3.79-17.29 \mathrm{pg} / \mathrm{mL} ; p<0.01)$ and on the ninetieth day from stroke onset (5.87: $2.30-10.58 \mathrm{vs}$ 10.9: 5.94-17.28 pg/mL; $p<0.01)$. There were no existing differences regarding hsCRP levels between groups (2.49: $0.11-9.82$ vs $4.44: 0.32-9.87 \mathrm{mg} / \mathrm{dL} ; p=0.30$ and $2.57: 0.11-2.57$ vs 2.83: $0.32-9.32 \mathrm{mg} / \mathrm{dL} ; p=0.75$, respectively). Patients with lacunar strokes were characterized by lower median of IL-6 (5.96: $2.87-13.0 \%$ vs $7.29: 2.30-17.28 ; p=<0.02)$ and hsCRP (2.25: $0.11-9.82$ vs $4.84: 0.35-9.87 ; p=0.01$ ) than those with nonlacunar infarctions. Multivariate analysis showed an impact of IL-6 on mRS measured on hospital dismission and after three months, regardless of their initial NIHSS, presence of hemorrhagic transformation and type 2 diabetes. No impact of hsCRP, lacunar etiology and patients' age on functional outcome existed.

Conclusion: Regardless of the stroke etiology, pretreatment of IL-6, but not of hsCRP levels, may help to predict functional outcome after IV-thrombolysis independently of symptom severity and stroke complications.

Keywords: inflammatory markers, ischemic stroke, thrombolysis, old age diseases

\section{Introduction}

Brain damage in acute phase of ischemic stroke (AIS) is a dynamic process inducing cellular-molecular immune response in the central nervous system (CNS) directed at the development of an inflammatory reaction. Ischemic stroke is the most common cause of disability in elderly people (over 65 years of age) and the third most common cause of death in the world. In the course of the brain damage, a number of biochemical mediators and inflammatory markers are released, ie cytokines, chemokines, pro-inflammatory enzymes and growth 
factors. A particularly rapid change in expression concerns pro-inflammatory cytokines. ${ }^{1,2}$ IL- 6 is believed to be a cytokine of particular importance in the acute stroke phase. It is responsible for inducement of synthesis, mainly in the liver, of acute-phase proteins (APPs): C-reactive proteins (CRP) and fibrinogen., ${ }^{3,4}$ Many authors point out that the increase in the concentration of IL- 6 in serum in the first day of stroke is associated with a deterioration in the functional status of patients ${ }^{5-7}$ and with a higher volume of ischemic lesions. ${ }^{8,9}$ It is also considered that IL-6 levels are a reliable prognostic factor in the acute phase of ischemic stroke. ${ }^{6,10,11}$ CRP levels, as a reagent of an acute immune response stimulated by IL-6, may also increase in acute stroke phase, although they may also occur in response to a wide spectrum of systemic inflammatory conditions. According to many authors, the increase in hsCRP levels is associated with poor prognosis, ${ }^{12-14}$ whereas other researchers suggest that CRP is not a prognostic factor in ischemic stroke. ${ }^{15,16}$

Intravenous (IV) thrombolytic therapy is now the most widely used form of effective AIS therapy. Among numerous prognostic factors of IV-thrombolysis, the most important are: the age of the patient, the severity of the neurological deficit, the onset-to-needle time and the presence of a large vessel occlusion. A number of publications also raise the issue of potential impact of CRP on the effectiveness of thrombolytic therapy, but there are no clear reports on IL-6 prognostic role in IV-thrombolysis. Moreover, the assessments of markers of inflammatory state presented in available reports were made in a wide time window or from the serum of blood taken only after thrombolytic therapy. Hence, the impact of inflammatory state on the prognosis of intravenous thrombolytic therapy still remains the object of controversy. ${ }^{17,18}$

Therefore, the purpose of our research was to assess serum IL-6 and hsCRP levels in the acute phase of ischemic stroke and to explore their role as an emerging marker in prediction of functional prognosis after thrombolytic therapy.

\section{Patients and Methods}

\section{Study Group}

We retrospectively evaluated the demographic and clinical data of 83 Caucasian patients with AIS treated with IV-thrombolysis (the median of patient age was 66, ranging from 35 to 92 years; $61.5 \%$ males) who were consecutively admitted to The Stroke Center of Department of
Neurology of the University Hospital No. 1 in Bydgoszcz, Poland from January to December 2018. The data was gathered only from patients treated $<4.5 \mathrm{~h}$ from stroke onset who gave a written informed consent. The IL-6 level determination was not routinely performed. Patients were subject to prolongation of the follow-up beyond the hospitalization period (up to three months). Those with combination therapy (intravenous plus endovascular therapy), concomitant myocardial infarction on the stroke onset and those who died within 24 hof stroke onset were excluded from the analysis. We have also excluded patients with prior history of stroke, chronic inflammatory and autoimmune diseases, neoplasms, steroid use and severe trauma or acute infections confirmed within \pm 3 days from hospital admission. The local ethics committee Collegium Medicum in Bydgoszcz, Nicolaus Copernicus University in Torun approved the protocol of the study (No KB 637/2016), in accordance with the Declaration of Helsinki.

The Stroke Center of the Department of Neurology of the University Hospital No. 1 in Bydgoszcz, Poland is recognized as a stroke unit according to the Polish national criteria and is equipped with appropriate monitoring and diagnostic facilities. ${ }^{19}$ The stroke unit provides a 24 -h stroke service seven days a week. All patients were examined at the time of admission by a stroke physician. The stroke was diagnosed on the basis of the ICD 10 criteria and it was confirmed on discharge by clinical evaluation and neuroimaging. Cerebral thrombolysis with the intravenous infusion of recombinant tissue plasminogen activator (rt-PA) was administered according to current guidelines. ${ }^{18,20}$ Computed tomography (CT) scans were performed in all patients upon admission to hospital and between 22 and $36 \mathrm{~h}$ after IV-thrombolysis. In selected cases, additional $\mathrm{CT}$ scans or magnetic resonance imaging (MRI) were performed in order to confirm the diagnosis. Stroke classification was made with the TOAST criteria, confirmed with information on vascular territory of a stroke. The severity of stroke symptoms was assessed using the National Institutes of Health Stroke Scale (NIHSS). Functional stroke outcomes were measured using the modified Rankin scale (mRS) on dismission and on the ninetieth day from the stroke onset. A favorable outcome was defined as mRS score $\leq 2$ points, while an unfavorable outcome was defined as an $\mathrm{mRS}$ of 3-6 points.

Comorbidities were diagnosed according to current guidelines: arterial hypertension with recommendations of the European Society of Cardiology (ESC); diabetes mellitus according to the criteria of the American 
Diabetes Association; dyslipidemia according to the ESC recommendations (Guidelines for the Management of Dyslipidemias), impaired renal function with eGFR $<60 \mathrm{~mL} / \mathrm{min} / 1.73 \mathrm{~m}^{2}$ and hyperuricemia with uremic acid serum concentration $>6.0 \mathrm{mg} / \mathrm{dL}$ or prior use of uremic acid lowering medication. The degrees of stenosis of the common carotid artery and/or internal carotid artery were assessed according to the criteria of Polish Section of Neurosonology. ${ }^{21}$

\section{Biochemical Testing}

All biochemical samples were routinely taken at the time of admission and the samples for biochemical analyses were obtained before starting IV-thrombolysis, within $4.5 \mathrm{~h}$ from the symptom onset. Samples of venous blood were taken from the cubital vein to $5 \mathrm{~mL}$ tubes with a coagulation activator and a gel separator. Blood for serum was left to clot and then centrifuged (3000 $\mathrm{g}$ for $15 \mathrm{~min}$ ) and separated into portions for Eppendorf tubes, where they were stored at $-80^{\circ} \mathrm{C}$ until designation of hsCRP and IL- 6 was performed.

ELISA enzyme immunoassays from BioVender, Brno, the Czech Republic and Diaclone, Besancon cedex, France were used to determine the concentration respectively of hsCRP and IL-6. All analyses were performed in accordance with the manufacturer's instructions and guidelines with a normal value for hsCRP below $3 \mu \mathrm{g} / \mathrm{mL}$.

\section{Statistical Methods}

This study was based on a retrospective data analysis. Univariate analysis and logistic regression were performed with STATISTICA v. 9.1 (Dell Inc., Round Rock, TX, USA). All continuous variables were tested for a normal distribution and equality of variance. Because of the nonnormality of the variables, nonparametric Mann-Whitney $U$-tests were used to perform the univariate analysis of the continuous variables and Spearman's rank test was used to evaluate correlations. Categorical data was compared using chi-squared tests. $P$ values $<0.05$ were considered statistically significant. The multivariate analysis was performed using generalized linear regression models. Factors identified in univariate analysis with a $p$ value $<0.05$ were included in the multivariate model. The results of the logistic regression models were presented as $p$ and OR with CI.

\section{Results}

Favorable outcome after IV-thrombolysis, defined as mRS 0-2 points, was diagnosed on hospital dismission in $60(72 \%)$ patients and in 62 (75\%) after 90 days from the stroke onset; $8(9.5 \%)$ patients died within 90 days of stroke onset. A previous history of hypertension was detected in $69(82.1 \%)$ patients, diabetes mellitus in $35(41.7 \%)$; coronary heart disease in $16(19 \%)$, carotid stenosis $>50 \%$ in $10(11.9 \%)$, atrial fibrillation (chronic or paroxysmal) in $11(13.1 \%)$, hyperlipidemia in $34(40.5 \%)$, chronic renal disease in $3(3.6 \%)$, hyperuricemia in $5(5.9 \%)$. Prior to stroke onset $28(33.3 \%)$ patients were on statins, $30(35.7 \%)$ on antiplatelet and one (1.2\%) was on anticoagulant therapy.

Medians of IL-6 serum concentration prior to initiation of IV-thrombolysis were lower in subgroup of patients with favorable than in those with unfavorable functional outcome gained, both at hospital dismission (5.92: 2.30-7.71 vs $9.463 .79-17.29 \mathrm{pg} / \mathrm{mL} ; p<0.01)$ and after 90 days from stroke onset (5.87: $2.30-10.58$ vs 10.9: 5.94-17.28 $\mathrm{pg} / \mathrm{mL} ; p<0.01$ ) (Figure 1).

There were no differences regarding hsCRP levels between groups of patients with favorable and unfavorable functional outcome assessed both on dismission (2.49: $0.11-9.82$ vs $4.44: 0.32-9.87 \mathrm{mg} / \mathrm{dL} ; p=0.30$ ) and after the ninetieth day from stroke onset (2.57: $0.11-2.57$ vs 2.83 : 0.32-9.32 mg/dL; $p=0.75$; respectively). Patients with a favorable outcome were also younger, had lower NIHSS score on admission, lower incidence of diabetes mellitus, post-stroke infections and hemorrhagic transformations and higher presence of lacunar etiology of stroke than those with an unfavorable outcome (Table 1).

There were significant correlations between serum IL-6 concentrations and NIHSS scores both, on hospital admission, and dismission from the hospital and mRS scores assessed on dismission and on the ninetieth day from the stroke onset. No correlations between hsCRP and NIHSS or mRS scores existed (Table 2). There was a correlation between NIHSS scores gained on hospital admission and dismission from the hospital $(\mathrm{R}=0.80 ; p<0.01)$. Serum IL-6 concentration was correlated with serum hsCRP concentrations $(\mathrm{R}=0.34 ; p<0.01)$. Subgroup of $52(61.9 \%)$ patients with lacunar strokes were characterized by lower median of IL-6 (5.96: 2.87-13.0\% vs 7.29: 2.30-17.28; $p=<0.02$ ) and hsCRP (2.25: $0.11-9.82$ vs 4.84: 0.35-9.87; $p=0.01$ ) than those with nonlacunar infarctions. There were no differences regarding both, IL-6 and hsCRP levels between subgroups of patients distinguished on the basis of significant artery stenosis (6.29: $2.30-13.97$ vs 7.89: $3.71-17.28 ; \quad p=0.10$ and $2.49: 0.11-9.87$ vs 3.02 : $0.35-8.67 ; p=0.74$; respectively) or presence of hemorrhagic transformation (7.29: $5.94-10.87$ vs 6.30 : $2.30-17.28$; 

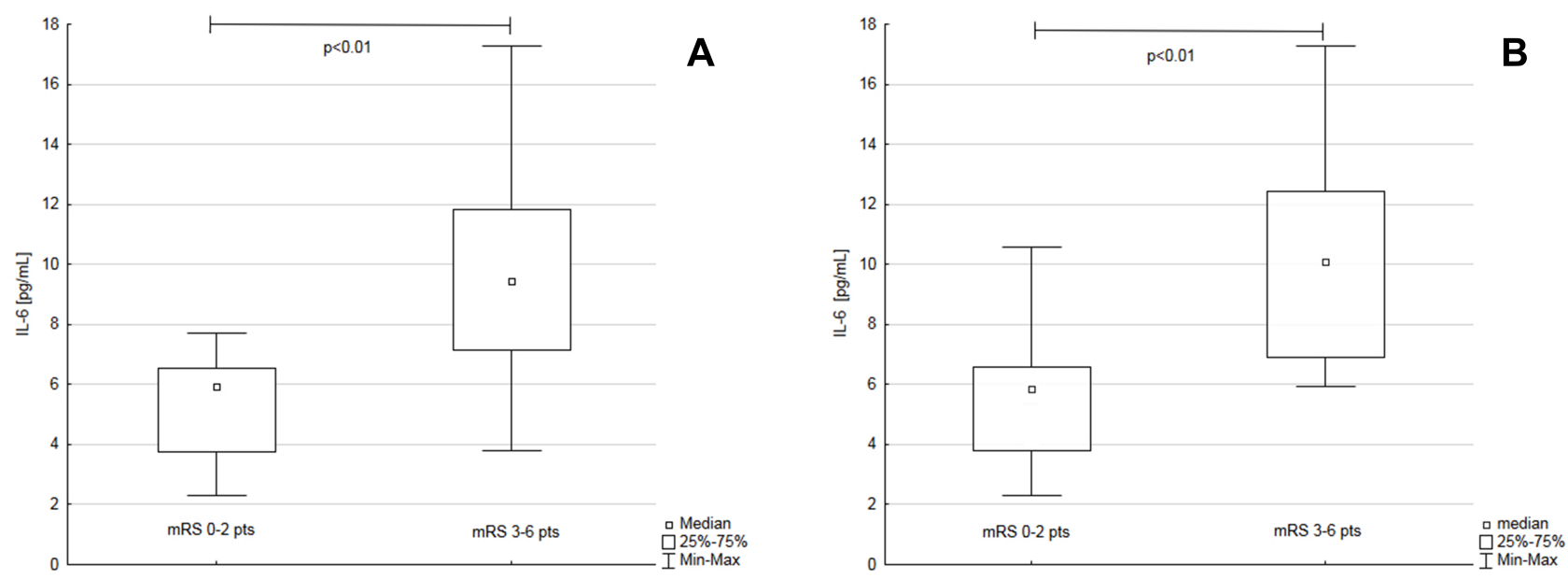

Figure I Pretreatment IL-6 serum concentrations in patients with and without favorable functional outcome on dismission (A) and on the ninetieth day (B) from the stroke onset.

$p=0.16$ and $4.8: 0.44-6.67$ vs $2.65: 0.11-9.87 ; p=0.68$; respectively). Patients with statins therapy prior to stroke were characterized by lower hsCRP levels, (1.98: $0.35-8.88$ vs $3.47: 0.11-9.87 ; p=0.03$ ), but there was no difference regarding IL-6 between subgroups of patients with and without statins therapy prior to stroke onset (6.38: $2.87-17.28$ vs 6.35: $2.30-16.89 ; p=0.98$ ). There were no correlations between age and IL-6 and hsCRP, both in patients with favorable $(\mathrm{R}=0.15, p=0.32$ and $\mathrm{R}=0.09, p=0.48$; respectively) and unfavorable outcome $(\mathrm{R}=0.14, p=0.58$ and $\mathrm{R}=-0.33, p=0.13$; respectively).

Multivariate analysis showed independent impact of IL-6, NIHSS score on admission on patients' functional outcome, assessed both on dismission from the hospital and on the ninetieth day from the stroke onset and additional impact of hemorrhagic transformation and type 2 diabetes on three-month outcome. No impacts of hsCRP, lacunar etiology and patients' age on long-term outcome were found. Presence of post-stroke infection showed an impact on short and long-term outcome in multivariate models with hsCRP, but no IL-6 as confounder (Table 3).

Pretreatment of IL-6 serum concentration showed good sensitivity and specificity for prediction of patients' outcome, both on dismission from the hospital $(\mathrm{AUC}=0.87)$ and within 90 days from the stroke onset $(\mathrm{AUC}=0.88)$ (Figure 2$)$.

\section{Discussion}

Numerous evidence suggests that inflammatory reaction is one of the main factors in the course of pathophysiological processes occurring during the acute phase of ischemic stroke. ${ }^{2,16}$ Therefore, pro-inflammatory cytokine activity can be important for the effectiveness of AIS patients' therapy.

Our study showed that higher IL-6 levels in the acute stroke phase were associated with worse early and longterm prognosis of patients treated with IV-thrombolysis. We found a relationship between IL-6 levels in the subacute AIS and the severity of the neurological deficit. Hence, we have proved that IL-6 is a prognostic factor in thrombolytic therapy of AIS. Similarly, other researchers showed that high levels of IL-6 in plasma in stroke patients were correlated with poor prognosis, ${ }^{6,22,23}$ expansive ischemic lesions. ${ }^{9,23-25}$ However, it should be emphasized that in most of those reports the IL- 6 levels were marked within $12-72 \mathrm{~h}$ of the acute stroke onset, while we have shown that the increase in IL-6 levels already in the first $4.5 \mathrm{~h}$ from onset of symptoms have a significant impact on short-term and distant prognosis of cerebral thrombolysis. Pusch et al found a similar relationship between early increase of IL-6 and outcome in AIS. ${ }^{26}$

According to our best knowledge, our study is one of the first to determine the effect of IL-6 serum levels marked before starting treatment on functional outcome after cerebral IV-thrombolysis. Because we have also shown a significant relationship between IL-6 and a functional outcome in a group of patients without prior infection and chronic inflammatory or cancer diseases, we believe that IL-6 is an inflammatory marker of acute ischemic brain injury, but not a chronic inflammatory state. In previous reports from other authors, IL-6 elevation has been often associated with a poor functional result, but it was not clear whether this was an independent effect or confounder of 
Table I The Clinical Characteristics of the Subgroups of Stroke Patients with a Favorable and an Unfavorable Outcome After IVThrombolysis

\begin{tabular}{|c|c|c|c|}
\hline \multirow[t]{3}{*}{ Clinical Parameters } & \multicolumn{3}{|c|}{ Outcome at 90 Days } \\
\hline & \multirow{2}{*}{$\begin{array}{l}\text { Favorable } \\
\text { (mRS 0-2 pts) }\end{array}$} & \multirow{2}{*}{$\begin{array}{l}\text { Unfavorable } \\
\text { (mRS 3-6 pts) }\end{array}$} & \multirow[t]{2}{*}{$\mathbf{p}$} \\
\hline & & & \\
\hline$n(\%)$ & $62(75)$ & $21(25)$ & - \\
\hline Age (mean; min-max) years & $65(25-88)$ & $70(46-92)$ & 0.02 \\
\hline Gender (male) n (\%) & $38(6 \mid .3)$ & $13(61.9)$ & 0.96 \\
\hline Baseline NIHSS median (range) points & $4.0(1-10)$ & $\mid 2.5(5-2 \mid)$ & $<0.01$ \\
\hline BMI median (IQR) kg/m² & $26.3(17.9-42.5)$ & $26.6(20.0-34.6)$ & 0.47 \\
\hline Onset-to-needletime median;(range) $\mathrm{min}$ & $207(55-270)$ & $195(75-270)$ & 0.38 \\
\hline SBP on admission median (range) $\mathrm{mmHg}$ & I5I (109-204) & $160(120-220)$ & 0.32 \\
\hline DBP on admission median (range) $\mathrm{mmHg}$ & $83(60-112)$ & $84(62-130)$ & 0.72 \\
\hline Heart rate on admission median (range) beats/min & $75(58-105)$ & $74(60-120)$ & 0.65 \\
\hline Hyperlipidemia n (\%) & $24(38.7)$ & $10(47.6)$ & 0.47 \\
\hline Hyperuricemia n (\%) & $5(8.6)$ & 0 & 0.18 \\
\hline Impaired renal function $\mathrm{n}(\%)$ & $2(3.2)$ & I (4.7) & 0.74 \\
\hline Diabetes mellitus n (\%) & $22(25.5)$ & $13(61.9)$ & 0.03 \\
\hline Current smoking n (\%) & $22(35.5)$ & $5(23.8)$ & 0.32 \\
\hline Arterial hypertension n (\%) & $50(80.6)$ & $18(85.7)$ & 0.60 \\
\hline Coronary heart disease n (\%) & II (I7.7) & $5(23.8)$ & 0.54 \\
\hline Carotid stenosis >50\% n (\%) & $6(9.7)$ & $4(21.0)$ & 0.19 \\
\hline Atrial fibrillation $n(\%)$ & $6(9.7)$ & $5(23.8)$ & 0.10 \\
\hline Statin therapy before stroke $\mathrm{n}(\%)$ & $20(32.3)$ & $8(38.1)$ & 0.66 \\
\hline Antiplatelet therapy before stroke $\mathrm{n}(\%)$ & $20(32.8)$ & $10(47.6)$ & 0.70 \\
\hline Anticoagulant therapy before stroke $\mathrm{n}(\%)$ & $\mathrm{I}(\mathrm{I} .6)$ & 0 & 0.23 \\
\hline Lacunar etiology of stroke $\mathrm{n}(\%)$ & $43(69.3)$ & $8(38.1)$ & 0.01 \\
\hline Hemorrhagic transformation $n(\%)$ & I ( 1.6$)$ & $6(28.6)$ & $<0.01$ \\
\hline Post-stroke infection (>3 day from stroke onset) $n$ (\%) & $3(4.8)$ & $9(42.8)$ & $<0.01$ \\
\hline $\mathrm{mRS}$ at the eventh day median (range) pts & $0(0-4)$ & $4(0-6)$ & $<0.01$ \\
\hline $\mathrm{mRS}$ at the ninetieth day median;(range) pts & $0(0-2)$ & $5(3-6)$ & $<0.01$ \\
\hline hsCRP median (range) $\mu \mathrm{g} / \mathrm{mL}$ & $2.57(0.11-2.57)$ & $2.83(0.32-9.32)$ & 0.75 \\
\hline IL-6 median (range) pg/mL & $5.87(2.30-10.58)$ & $10.09(5.94-17.28)$ & $<0.01$ \\
\hline
\end{tabular}

Abbreviations: mRS, modified Rankin Scale; NIHSS, National Institutes of Health Stroke Scale; SBP, systolic blood pressure; DBP, diastolic blood pressure; CRP, C-reactive protein; IL-6, interleukin-6; IQR, interquartile range $\left(Q_{1}-Q_{3}\right)$.

concomitant infection. ${ }^{5,27}$ Interestingly, we found a positive correlation between IL-6 and hsCRP levels in AIS patients, whereas, similarly to other authors, we have not confirmed previous reports showing a relationship between hsCRP and the prognosis of thrombolytic therapy. This may be due to some limitations, as patients with mild strokes participated in the study. That is why it seems IL-6 rather than CRP is produced in response to cerebral ischemia and is a strong stimulus for the production of acute phase proteins in the liver. $^{28-32}$ Only patients who had no signs of infection within \pm 3 days of developing the disease were included in our study. In view of the presence of a positive correlation between these parameters, this fact may indicate that hsCRP is not only an indicator of infection in the subacute phase, but similarly to Molnar et $\mathrm{al}^{33}$ it could also be a marker of vascular damage in the course of atherosclerosis. Regardless of that, like other authors, we showed that stroke associated infection (more than three days after the disease) had an impact on the short and long-term prognosis, and this was regardless of the value of hsCRP and IL- 6 determined at the time of admission. ${ }^{34}$

Similarly to other authors, we have showed lower serum IL-6 values in patients with lacunar stroke caused by cerebral microangiopathy than in patients with other AIS etiologies. ${ }^{35,36}$ This fact, as well as the presence of correlation between IL-6 levels measured $<4.5 \mathrm{~h}$ from symptoms onset and the stroke symptoms' severity (NIHSS score), both on hospital admission and discharge, 
Table 2 Correlations Between Biochemical Markers and Onset-to-Needle Time and the Severity of Neurological on Admission and Functional Outcome on the seventh and the ninetieth Day from Stroke Onset

\begin{tabular}{|l|l|l|}
\hline Factors & $\mathrm{IL}-6 \mathbf{~ p g} / \mathbf{m L}$ & hsCRP $\boldsymbol{\mu g} / \mathbf{m L}$ \\
\hline Onset-to-needle time, min & $\mathrm{R}=-0.09 ; p=0.47$ & $\mathrm{R}=-0.01 ; p=0.86$ \\
NIHSS score on admission, pts & $\mathrm{R}=0.42 ; p<0.0 \mathrm{I}$ & $\mathrm{R}=0.1 \mathrm{I} ; p=0.32$ \\
NIHSS score on discharge, pts & $\mathrm{R}=0.58 ; p<0.0 \mathrm{I}$ & $\mathrm{R}=0.22 ; p=0.05$ \\
mRS on dismission, pts & $\mathrm{R}=0.59 ; p<0.0 \mathrm{I}$ & $\mathrm{R}=0.22 ; p=0.04$ \\
mRS at the ninetieth day from stroke onset, pts & $\mathrm{R}=0.66 ; p<0.0 \mathrm{I}$ & $\mathrm{R}=0.91 ; p=0.09$ \\
\hline
\end{tabular}

Abbreviations: mRS, modified Rankin Scale; NIHSS, National Institutes of Health Stroke Scale; CRP, C-reactive protein; IL-6, interleukin-6.

Table 3 Multivariate Logistic Regression Models Showing Factors Associated with Functional Outcome on Dismission and on the 90th Day from Stroke Onset (Model I: IL-6 as Confounder, Model 2: hsCRP as a Confounder)

\begin{tabular}{|c|c|c|c|c|c|c|c|c|}
\hline \multirow[t]{3}{*}{ Factors } & \multicolumn{4}{|c|}{ Favorable Outcome on Dismission } & \multicolumn{4}{|c|}{ Favorable Outcome on ninetieth Day } \\
\hline & \multicolumn{2}{|l|}{ Model I } & \multicolumn{2}{|l|}{ Model 2} & \multicolumn{2}{|l|}{ Model I } & \multicolumn{2}{|l|}{ Model 2} \\
\hline & OR $(95 \% \mathrm{Cl})$ & $p$ & OR $(95 \% \mathrm{Cl})$ & $p$ & OR $(95 \% \mathrm{Cl})$ & $p$ & OR $(95 \% \mathrm{Cl})$ & $p$ \\
\hline $\mathrm{LACl}$ & $3.04(0.47-19.54)$ & 0.23 & $3.39(0.28-39.70)$ & 0.33 & $0.01(<0.01-2.63)$ & 0.11 & $0.03(0.01-2.18)$ & 0.11 \\
\hline Type 2 diabetes & $0.39(0.06-2.55)$ & 0.32 & $0.61(0.05-6.73)$ & 0.69 & $0.02(<0.01-0.72)$ & 0.03 & $0.04(0.01-1.02)$ & 0.04 \\
\hline Age, years & $1.04(0.96-1.12)$ & 0.34 & $1.01(0.91-1.13)$ & 0.75 & $1.07(0.96-1.19)$ & 0.19 & $1.06(0.91-1.25)$ & $0.4 \mathrm{I}$ \\
\hline Hemorrhagic transformation & $0.15(0.01-2.53)$ & 0.19 & $0.03(0.01-1.70)$ & 0.09 & $0.0 \mathrm{I}(<0.0 \mathrm{I}-0.3 \mathrm{I})$ & 0.01 & $0.01(<0.01-0.2 \mathrm{I})$ & 0.02 \\
\hline Post-stroke infection & $0.58(0.01-22.50)$ & 0.77 & $0.03(0.01-0.89)$ & 0.04 & $0.67(0.02-24.10)$ & 0.82 & $0.01(<0.01-0.57)$ & 0.03 \\
\hline NIHSS on admission, pts & - & - & $2.55(1.43-4.54)$ & $<0.01$ & - & - & $4.76(1.46-15.50)$ & $<0.01$ \\
\hline hsCRP, $\mu \mathrm{g} / \mathrm{mL}$ & - & - & $1.13(0.7 \mathrm{I}-1.80)$ & 0.59 & - & - & $0.66(0.30-1.43)$ & 0.29 \\
\hline IL-6, pg/mL & $2.14(1.19-3.84)$ & 0.01 & - & - & $5.85(1.46-23.48)$ & 0.01 & - & - \\
\hline
\end{tabular}

Abbreviations: LACI, lacunar cerebral infarction; CRP, C-reactive protein; IL-6, interleukin-6; NIHSS, National Institutes of Health Stroke Scale.

prove that IL-6 may be considered as a potential marker of the extent of ischemic brain injury and its further clinical consequences. ${ }^{37}$ However, unlike previous publications, we have not demonstrated the relationship between the presence of atherosclerosis changes in the carotid arteries and the level of IL- $6 .^{38,39}$ This fact may be explained by the limited number of the group (type I error).

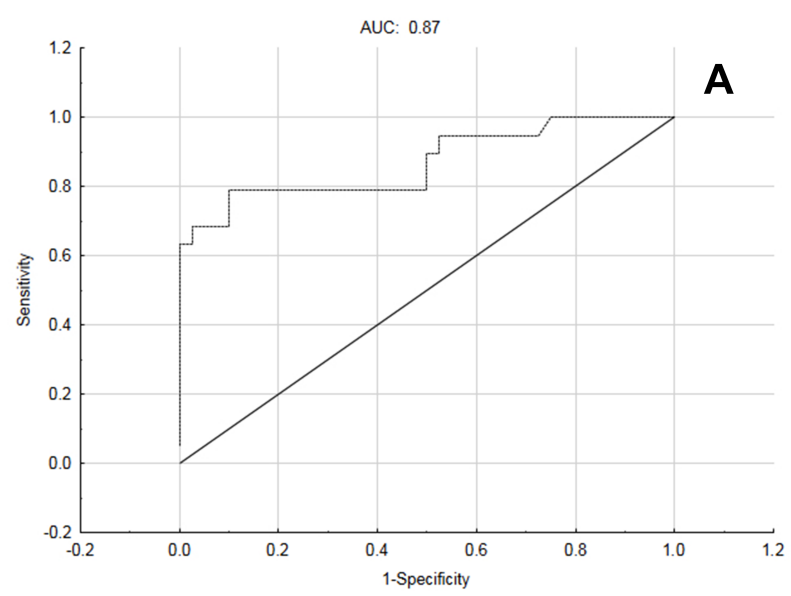

It has been noted that IL- 6 exacerbates the deterioration of brain damage, counteracts the proliferation of nerve stem cells, but has not always been found despite its high concentration in the early stages of stroke, the effect on long-term and short-term prognosis or the degree of neurological and functional deficit in AIS patients. ${ }^{10,24,40,41}$ Others also report its neuroprotective role. ${ }^{42,43}$

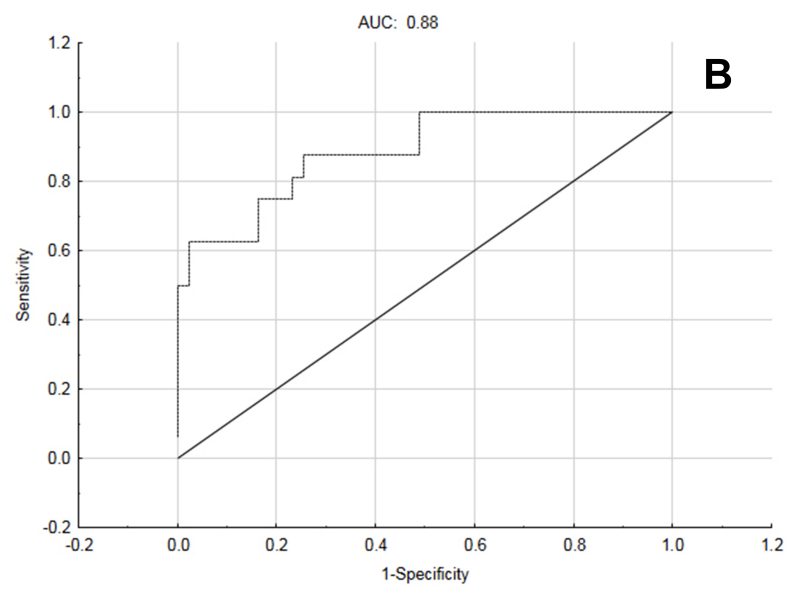

Figure 2 ROC curves showing sensitivity and specificity of IL-6 measurements for good functional outcome: (A) on dismission (cut-off point $\leq 7.06$ pg/mL, sensitivity $90 \%$, specificity $78.9 \%$ ), (B) on the ninetieth day from the stroke onset (cut-off point $\leq 6.5 \mathrm{I} \mathrm{pg/mL}$, sensitivity $74.4 \%$, specificity $87.5 \%$ ). 
Regardless of the demonstrated impact of proinflammatory factors on patient prognosis, we have also shown a significant impact of the severity of the neurological deficit assessed with NIHSS scale for short and longterm prognosis of AIS patients treated IV-thrombolysis. This is consistent with previous literature reports. ${ }^{44-47}$ Moreover, similar to other authors, we found a weak, but potentially negative effect of diabetes on the long-term prognosis of thrombolytic therapy in patients with light and moderate symptoms of the disease. That may indicate a certain caution regarding thrombolytic stroke therapy in patients with diabetes mellitus, regardless current liberalization of the guidelines. ${ }^{18,48}$ We have also found a negative effect of hemorrhagic transformation on the long-term prognosis of thrombolytic therapy, as already pointed out by some authors. 49

Our report has some limitations. Among the most important is the fact that this is a single-center study research, which has reduced the number of examined subjects. The need to give informed consent for the additional blood sampling for the purposes of cytokines markings resulted in the exclusion of patients with aphasia or with impaired awareness. Consequently, we recruited mainly patients with slight and moderate neurological deficit. Due to both the abovementioned limitations, and results of the contradictory results of other reports, we believe that studies of the effects of IL-6 levels on the prognosis of thrombolytic therapy should be continued on a larger population, as a multicenter study.

\section{Conclusion}

In the study, we showed that IL-6 could have potential prognostic significance for the success of thrombolytic therapy, while we did not show such relationship in the case of hsCRP.

IL-6 may have potential practical applications as a prognostic parameter for the patient's acute phase of stroke, but nevertheless further clinical trials are needed in more patients with acute ischemic stroke. Studies should also be carried out in many centers involving the population of patients not only with mild to moderate neurological deficits.

It may also be interesting to consider the use of immunomodulatory therapy aimed at limiting the early inflammatory response in stroke by inhibiting IL-6. The effectiveness of this form of therapy using the IL-6 receptor inhibiting antibody, tocilizumab has already been observed in the treatment of certain diseases. ${ }^{50}$ It may be possible that this may also be an important clue in the design of novel forms of AIS therapy in the brain. An example of this is the report by Smith et $\mathrm{al}^{51}$ suggesting that administration of an IL-1 (IL-1R) receptor antagonist that induces IL-6 may lead to an improvement in the clinical outcome of treating the acute phase of ischemic stroke.

\section{Acknowledgment}

The study was financed by Collegium Medicum in Bydgoszcz, Nicolaus Copernicus University in Torun.

\section{Disclosure}

The authors report no conflicts of interest in this work.

\section{References}

1. Mizuma A, Yenari MA. Anti-inflammatory targets for the treatment of reperfusion injury in stroke. Front Neurol. 2017;8:467-487. doi:10.3389/fneur.2017.00467

2. Vidale S, Consoli A, Arnaboldi M, Consoli D. Postischemic inflammation in acute stroke. J Clin Neurol. 2017;13(1):1-9. doi:10.3988/ jcn.2017.13.1.1

3. Matsumoto J, Dohgu S, Takata F, et al. TNF- $\alpha$-sensitive brain pericytes activate microglia by releasing IL- 6 through cooperation between IjB-NFjB and JAK-STAT3 pathways. Brain Res. 2018;1692:34-44. doi:10.1016/j.brainres.2018.04.023

4. Ramiro L, Simats A, García-Berrocoso T, Montaner J. Inflammatory molecules might become both biomarkers and therapeutic targets for stroke management. Ther Adv Neurol Disord. 2018;11:1-24. doi:10.1177/1756286418789340

5. Bustamante A, Sobrino T, Giralt D, et al. Prognostic value of blood interleukin-6 in the prediction of functional outcome after stroke: a systematic review and meta-analysis. $J$ Neuroimmunol. 2014;15:215-224. doi:10.1016/j.jneuroim.2014.07.015

6. Choudhary S, Chowdhur D, Mishra TK, et al. Temporal profile of serum levels of IL-6 in acute ischemic stroke and its relationship with stroke severity and outcome in indian population. Int J Intg Med Sci. 2018;5:555-560

7. Dobrucka-Głowacka A, Sarecka-Hujar B, Raczkiewicz D, et al. Interleukin-6 in cardiovascular disorders. Eur J Med Technol. 2018;3:23-30.

8. Martínez-Sánchez P, Gutiérrez-Fernández $\mathrm{M}$, Fuentes $\mathrm{B}$, et al. Biochemical and inflammatory biomarkers in ischemic stroke: translational study between humans and two experimental rat models. J Transl Med. 2014;220:1-13.

9. Hotter B, Hoffmann S, Ulm L, et al. 1C IL-6 Plasma levels correlate with cerebral perfusion deficits and infarct sizes in stroke patients without associated infections. Front Neurol. 2019;10:1-8. doi:10.3389/fneur.2019.00083

10. Whiteley W, Chong WL, Sengupta A, et al. Blood markers for the prognosis of ischemic stroke: a systematic review. Stroke. 2009;40 (5):380-389. doi:10.1161/STROKEAHA.108.528752

11. Park SY, Kim J, Kim OJ, et al. Predictive value of circulating interleu kin- 6 and heart-type fatty acid binding protein for three months clinical outcome in acute cerebral infarction: multiple blood markers profiling study. Crit Care. 2013;17(2):1-9. doi:10.1186/cc12564

12. Song IU, Kim JS, Kim YI, et al. Relationship between high-sensitivity $\mathrm{C}$-reactive protein and clinical functional outcome after acute ischemic stroke in a Korean population. Cerebrovasc Dis. 2009;28:545-550. doi:10.1159/000247597

13. Song IU, Kim YD, Kim JS, et al. Can high sensitivity C-reactive protein and plasma homocysteine levels independently predict the prognosis of patients with functional disability after first-ever ischemic stroke? Eur Neurol. 2010;64:304-310. doi:10.1159/ 000321415 
14. Bunevicius A, Kazlauskas H, Raskauskiene N, et al. Role of N-terminal pro-B-type natriuretic peptide, high-sensitivity $\mathrm{C}$-reactive protein, and inteleukin-6 in predicting a poor outcome after a stroke. Neuroimmunomodulation. 2015;22(6):365-372. doi:10.1159/000381218

15. Ozkan AK, Yemisci OU, Saracgil Cosar SN, et al. Can high-sensitivity $\mathrm{C}$-reactive protein and ferritin predict functional outcome in acute ischemic stroke? A prospective study. Top Stroke Rehabil. 2013;20(6):528-536. doi:10.1310/tsr2006-528

16. Bustamante A, Simats A, Vilar-Bergua A, et al. Blood/brain biomarkers of inflammation after stroke and their association with outcome: from C-reactive protein to damage-associated molecular patterns. Neurotherapeutics. 2016;13(4):671-684. doi:10.1007/s13311-0160470-2

17. Simats A, García-Berrocoso T, Montaner J. Neuroinflammatory biomarkers: from stroke diagnosis and prognosis to therapy. Biochim Biophys Acta. 2016;1862:411-424. doi:10.1016/j.bbadis.2015.10.025

18. Powers WJ, Rabinstein AA, Ackerson T, et al. Guidelines for the early management of patients with acute ischemic stroke: a guideline for healthcare professionals from the American Heart Association/ American stroke association. Stroke. 2018;2018(49):46-110.

19. Błażejewska-Hyżorek B, Czernuszenko A, Członkowska A, et al. Wytyczne postępowania w udarze mózgu. Polski Przegląd Neurologiczny. 2019;15:1-156. doi:10.5603/PPN.2019.0001

20. Ahmed N, Audebert H, Turc G, et al. Consensus statements and recommendations from the ESO-Karolinska stroke update conference, Stockholm 11-13 November 2018. Eur Stroke J. 2019;4 (4):307-317. doi:10.1177/2396987319863606

21. Wojczal J, Tomczyk T, Luchowski P, et al. Standards in neurosonology. Part I. J Utrason. 2015;15(62):307-317. doi:10.15557/ JoU.2015.0027

22. Shenhar-Tsarfaty S, Ben AE, Bova I, et al. Interleukin-6 as an early predictor for one-year survival following an ischaemic stroke/transient ischaemic attack. Int J Stroke. 2010;5:16-20. doi:10.1111/j.17474949.2009.00396.x

23. Li X, Lin S, Chen X, et al. The prognostic value of serum cytokines in patients with acute ischemic stroke. Aging Dis. 2019;10 (3):544-556. doi:10.14336/AD.2018.0820

24. Smith CJ, Emsley HC, Gavin CM, et al. Peak plasma interleukin-6 and other peripheral markers of inflammation in the first week of ischaemic stroke correlate with brain infarct volume, stroke severity and long-term outcome. BMC Neurol. 2004;4:1-8. doi:10.1186/14712377-4-1

25. Fahmi RM, Elsaid AF. Infarction size, interleukin-6, and their interaction are predictors of short-term stroke outcome in young egyptian adults. $J$ Stroke Cerebrovasc Dis. 2016;25(10):2475-2481. doi:10.1016/j.jstrokecerebrovasdis.2016.06.021

26. Pusch G, Debrabant B, Molnar T, et al. Early dynamics of P-selectin and interleukin 6 predicts outcomes in ischemic stroke. J Stroke Cerebrovasc Dis. 2015;24(8):1938-1947. doi:10.1016/j.jstrokecerebrovasdis.2015. 05.005

27. Emsley HC, Smith CJ, Gavin CM, et al. An early and sustained peripheral inflammatory response in acute ischaemic stroke: relationships with infection and atherosclerosis. $J$ Neuroimmunol. 2003;139:93-101. doi:10.1016/S0165-5728(03)00134-6

28. Cao JJ, Thach C, Manolio TA, et al. C-reactive protein, carotid intima-media thickness, and incidence of ischemic stroke in the Elderly. The cardiovascular health study. Circulation. 2003;108:166-170. doi:10.1161/01.CIR.0000079160.07364.6A

29. Montaner J, Fernandez-Cadenas I, Molina CA, et al. Poststroke C-reactive protein is a powerful prognostic tool among candidates for thrombolysis. Stroke. 2006;37:1205-1210. doi:10.1161/01. STR.0000217744.89208.4e

30. Topakian R, Strasak AM, Nussbaumer K, et al. Prognostic value of admission C-reactive protein in stroke patients undergoing iv thrombolysis. $J$ Neurol. 2008;255(8):1190-1196. doi:10.1007/ s00415-008-0866-y
31. Karlinski M, Bembenek J, Grabska K, et al. Routine serum C-reactive protein and stroke outcome after intravenous thrombolysis. Acta Neurol Scand. 2014;130(5):305-311. doi:10. 1111/ane. 12227

32. Gill D, Sivakumaran P, Wilding P, et al. Trends in C-reactive protein levels are associated with neurological change 24 hours after thro mbolysis for acute ischaemic stroke. Cerebrovasc Dis. 2016;25 (8):1966-1969. doi:10.1016/j.jstrokecerebrovasdis.2016.05.003

33. Molnar T, Papp V, Banati M, et al. Relationship between C-reactive protein and early activation of leukocytes indicated by leukocyte antisedimentation rate (LAR) in patients with acute cerebrovascular events. Clin Hemorheol Microcirc. 2010;44(3):183-192. doi:10.32 33/CH-2010-1273

34. Suda S, Aoki J, Shimoyama T, et al. Stroke-associated infection independently predicts 3-month poor functional outcome and mortality. $J$ Neurol. 2018;265(2):370-375. doi:10.1007/s00415-0178714-6

35. Tuttolomondo A, Di Sciacca R, Di Raimondo D, et al. Plasma levels of inflammatory and thrombotic/fibrinolytic markers in acute ischemic strokes. Relationship with TOAST subtype, outcome and infarct site. $J$ Neuroimmunol. 2009;215:84-89. doi:10.1016/j. jneuroim.2009.06.019

36. Tuttolomondo A, Di Raimondo D, Pecoraro R, et al. Inflammation in ischemic stroke subtypes. Curr Pharm Des. 2012;18:4289-4310. doi:10.2174/138161212802481200

37. Lasek-Bal A, Jedrzejowska-Szypulka H, Student S, et al. The importance of selected markers of inflammation and blood-brain barrier damage for short-term ischemic stroke prognosis. $J$ Physiol Pharmacol. 2019;70(2):209-217.

38. Koutouzis M, Rallidis LS, Peros G, et al. Serum interleukin-6 is elevated in symptomatic carotid bifurcation disease. Acta Neurol Scand. 2009;119(2):119-125. doi:10.1111/j.1600-0404.2008.01068.x

39. Puz P, Lasek-Bal A. Repeated measurements of serum concentrations of TNF-alpha, interleukin-6 and interleukin-10 in the evaluation of internal carotid artery stenosis progression. Atherosclerosis. 2017;263:97-103. doi:10.1016/j.atherosclerosis.2017.06.008

40. Whiteley W, Jackson C, Lewis S, et al. The association of circulating inflammatory markers with recurrent vascular events after stroke: a prospective cohort study. Stroke. 2011;42:10-16. doi:10.1161/ STROKEAHA.110.588954

41. Whiteley W, Wardlaw J, Dennis M, et al. The use of blood biomarkers to predict poor outcome after acute transient ischemic attack or ischemic stroke. Stroke. 2012;43:86-91. doi:10.1161/STROKEAHA. 111.634089

42. Gertz K, Kronenberg G, Kälin RE, et al. Essential role of interleukin-6 in post-stroke angiogenesis. Brain. 2012;135:1964-1980.

43. Sotgiu S, Zanda B, Marchetti B, et al. Inflammatory biomarkers in blood of patients with acute brain ischemia. Eur $J$ Neurol. 2006;13:505-513. doi:10.1111/j.1468-1331.2006.01280.x

44. Emberson J, Lees KR, Lyden P, et al. Effect of treatment delay, age, and stroke severity on the ef fects of intravenous thrombolysis with alteplase for acute ischaemic stroke: a meta-analysis of individual patient data from randomised trials. Lancet. 2014;384(9958):1929-1935. doi:10.1016/S0140-6736(14)60584-5

45. Demaerschalk BM, Kleindorfer DO, Adeoye OM, et al. Scientific rationale for the inclusion and exclusion criteria for intravenous alteplase in acute ischemic stroke: a statement for healthcare professionals from the American Heart Association/American Stroke Association. Stroke. 2016;47(2):581-641. doi:10.1161/STR.000000 0000000086

46. Åsberg S, Farahmand B, Hasvold P, et al. Non-cardioembolic TIA and ischemic stroke: implications of severity. Acta Neurol Scand. 2018;138(4):369-376. doi:10.1111/ane.12974

47. Kim DH, Lee DS, Nah HW, et al. Clinical and radiological factors associated with unfavorable outcome after intravenous thrombolysis in patients with mild ischemic stroke. BMC Neurol. 2018;30:1-8. 
48. Chen G, Wang X, Robinson TG, et al. Comparative effects of low-dose versus standard-dose alteplase in ischemic patients with prior stroke and/or diabetes mellitus: the ENCHANTED trial. J Neurol Sci. 2018;387(387):1-5. doi:10.1016/j.jns.2018.01.014

49. D'Amelio M, Terruso V, Famoso G, et al. Early and late mortality of spontaneous hemorrhagic transformation of ischemic stroke. J Stroke Cerebrovasc Dis. 2014;23:649-654. doi:10.1016/j.jstrokecerebrovasdis. 2013.06.005
50. Narazaki M, Kishimoto T. The two-faced Cytokine IL-6 in host defense and diseases. Int J Mol Sci. 2018;19(11):1-28. doi:10.3390/ ijms 19113528

51. Smith CJ, Hulme S, Vail A, et al. SCIL-STROKE (subcutaneous interleukin-1 receptor antagonist in ischemic stroke): a randomized controlled Phase 2 trial. Stroke. 2018;49(5):1210-1216. doi:10.1161/ STROKEAHA.118.020750

\section{Publish your work in this journal}

Clinical Interventions in Aging is an international, peer-reviewed journal focusing on evidence-based reports on the value or lack thereof of treatments intended to prevent or delay the onset of maladaptive correlates of aging in human beings. This journal is indexed on PubMed Central, MedLine, CAS, Scopus and the Elsevie
Bibliographic databases. The manuscript management system is completely online and includes a very quick and fair peer-review system, which is all easy to use. Visit http://www.dovepress.com/ testimonials.php to read real quotes from published authors.

Submit your manuscript here: https://www.dovepress.com/clinical-interventions-in-aging-journal 